\title{
Electron paramagnetic resonance and phosphorescence measurements of the triplet state of orotic acid and related pyrimidines
}

\author{
A. $\mathrm{H}_{\mathrm{AUG}}{ }^{*}$ and P. Douzou \\ Laboratoire de Biophysique, Muséum National d'Histoire Naturelle, Paris \\ (Z. Naturforschg. 20 b, 509-512 [1965] ; eingegangen am 25. Januar 1965)
}

\begin{abstract}
With orotic acid an EPR triplet state signal $(\Delta \mathrm{M}= \pm 2)$ was observed in a non-cracking rigid solvent at $77^{\circ} \mathrm{K}$. This triplet state, lying at $470 \mathrm{~m} \mu$, has a mean half-life of 0.2 sec and is characterized by a large zero-field splitting.

Excitation and phosphorescence spectra of related pyrimidines were measured, too. Besides uracil and cytosine, the excitation peak of these pyrimidines coincides with the large $\pi-\pi^{*}$ absorption peak. In the case of uracil and cytosine the excitation peak lies distinctly separated at the long wavelength side of the corresponding absorption peak. This probably indicates a $n-\pi^{*}$ excitation. The failure to record EPR triplet signals in these pyrimidines is attributed to large zero-field splitting.
\end{abstract}

The photochemistry of the DNA, the carrier of the genetic information, started with the discovery that thymine dimerizes ${ }^{1}$. Which electronically excited states are participating in photochemical reactions of biological purines and pyrimidines is rather unknown ${ }^{2}$. In the case of uracil and orotic acid BERENDs et al. ${ }^{3}$ found that paramagnetic molecules or ions inhibit the dimerization of these compounds in aqueous solution. Studying by the molecular orbital method the electronic structure of the pyrimidine bases undergoing photodimerization, Mantione and Pullman ${ }^{4}$ concluded that this photochemical reaction occurs via a triplet state. But up to now it was impossible to demonstrate uniquely that a triplet state is involved in a photochemical reaction such as dimerization.

Orotic acid seemed $t$, be a favorable compound to be investigated, because its dimerization reaction in aqueous solution shows a large oxygen effect ${ }^{3,5}$. To detect probable triplet states we used the methods of electron paramagnetic resonances, phosphorescence, and triplet-triplet energy transfer between different molecules, respectively.

To get some insight into the electronical evolution of this probable triplet state of orotic acid, related compounds were examined.

* Present address: Gates and Crellin Laboratories, California Institute of Technology, Pasadena/California.

1 R. Beukers and W. Berends, Biochim. biophysica Acta [Amsterdam] 41, 550 [1960].

2 M. Kasha, in: Light and Life, edited by W. D. McElroy and B. Glass (The John Hopkins University Press, Baltimore 1961) ; G. W. Robinson, in: Light and Life, edited by W. D. McElroy and B. Glass (The Johns Hopkins University Press, Baltimore, 1961).

\section{Experimental}

$E P R$ measurements were performed with a $100 \mathrm{kc}$ Varian 4502 spectrometer equipped with an optical transmission cavity accessory to irridiate the sample at $77^{\circ} \mathrm{K}$. Ultraviolet irradiations were carried out with an Osram HB 200 lamp. A water filter avoided heating up of the sample. $\Delta \mathrm{M}= \pm 2$ transitions for triplets ${ }^{6}$ were observed for randomly oriented molecules dissolved in a non-cracking glassy matrix (dimethyl formamide : ethanol : ether $=1: 1: 1.7, \mathrm{v} / \mathrm{v})$. The sample was contained in a quartz tube of $3 \mathrm{~mm}$ inner diameter. Before sealing off this quartz tube, oxygen was removed by flushing the solution with purified nitrogen gas following by evacuation up to $5 \times 10^{-4}$ Torr in a vacuum line.

An Aminco-Keirs spectrophosphorimeter was used to measure excitation and phosphorescence spectra at $77{ }^{\circ} \mathrm{K}$. The sample could be adjusted by screws for maximum intensity. The sample was contained in the same quartz tube described above. The output of the 1-P-28 photomultiplier was fed to a X-Y-recorder to graph excitation and phosphorescence spectra. Below $250 \mathrm{~m} \mu$ excitation spectra could not be recorded with accuracy. A camera mounted on a Tektronix oscilloscope measured the decay of the phosphorescent state.

Absorption spectra were determined with a Cary 15.

The compounds tested were purchased from Calbiochem Corporation (orotic acid, uracil, thymine, 5-hydroxymethyl cytosine, cytosine-5-carboxylic acid, 2-thiouracil-5-carboxylic acid, uracil-6-acetic acid, 5amino orotic acid, 5-nitro orotic acid, dihydro uracil,

3 R. Beukers, J. Yjlstra and W. Berends, Rec. trav. chim. Pays-Bas 78, 879, 883 [1959]; W. Berends and R. Beukers, Biochim. biophysica Acta [Amsterdam] 38, 573 [1960].

4 H. J. Mantione and B. Pullman, Biochim. biophysica Acta [Amsterdam] 91, 387 [1964].

5 A. HaUG, J. Amer. chem. Soc. 86, 3381 [1964].

6 M.S. De Groot and H. J. van der WaAls, J. molecular Phys. 3,190 [1960]. 
dihydro thymine, dihydro orotic acid), from Sigma Co. (orotic acid, cytosine, dihydro orotic acid, azathymine, bromouracil), from Aldrich Co. (orotic acid, 5-diazo uracil, isoorotic acid = uracil-5-carboxylic acid). Orotidine and $3-\mathrm{N}$-methyl thymidine were kindly supplied by Dr. A. M. Michelson, Paris, 5-methyl orotic acid was a gift from Dr. C. Mentzer, Paris. Prior to use, orotic acid and isoorotic acid were four times recrystallized from water, dihydro thymine was crystallized from ethanol after passing a column of $\mathrm{Al}_{2} \mathrm{O}_{3}$. There was no difference in the phosphorescence spectra between these purified compounds and the non-purified material.

Solvents (dimethyl formamide, ethanol, ether) were purchased from E. Merck. Ether was purified by passing through a column of $\mathrm{Al}_{2} \mathrm{O}_{3}$. Dimethyl formamide was vacuum distilled; with $\mathrm{AgNO}_{3}$ it did not give any change.

\section{Results}

EPR spectrum of orotic acid. The EPR spectrum of orotic acid in the rigid solvent (dimethyl-formamide : ethanol : ether $=1: 1: 1.7$ ) is graphed in Fig. 1. The position of this triplet signal at $\mathrm{H}_{\min }=$ $1232 \mathrm{G}$ was determined relative to the well known triplet signal of naphthalene at $\mathrm{H}_{\min }=1533 \mathrm{G}^{6}$. The difference in the magnetic field strength between orotic acid and naphthalene was measured with a Varian Fieldial V-FR 200. The half-width of the orotic acid triplet signal amounts to $37 \pm 2 \mathrm{G}$. Searching for $\Delta \mathrm{M}=1$ transitions was unsuccessful. Being at the maximum of the signal amplitude, switching off the irradiating light results in a decrease of the EPR signal up to zero. The EPR signal intensity of orotic acid at $77^{\circ} \mathrm{K}$ varies linearly with concentration between $3 \times 10^{-4}$ and $3 \times 10^{-3} \mathrm{M}$. Taking concentrations of the order of $10^{-2} \mathrm{M}$ results in precipitation of orotic acid after standing at room temperature for half a day. Therefore we did not use these concentrations for our measurements. To record the signal amplitude of the sample we had to work at the upper limit of the signal level, precise optical adjustment for the irradiation procedure was necessary, too. Enhancing the temperature of the sample in the microwave cavity from $77^{\circ} \mathrm{K}$ to about $95^{\circ} \mathrm{K}$, there is only a very small triplet signal left.

With all other compounds studied (Tab. 1), besides 5-diazo uracil, we were not able to find a triplet EPR signal. The diazo uracil triplet signal is positioned at $\mathrm{H}_{\min }=1360 \mathrm{G}$, having a halfwidth of $23 \mathrm{G}$.

Excitation and phosphorescence spectra. The experimentally determined values are plotted in Tab. 1 .

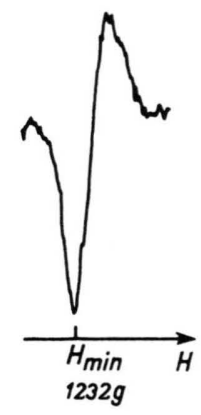

Fig. 1. EPR triplet signal $(\Delta \mathrm{M}= \pm 2)$ of orotic acid in the rigid solvent (dimethyl formamide: ethanol:ether $=1: 1: 1.7$ ) at $77^{\circ} \mathrm{K}$. Frequency $9208 \mathrm{Mc} / \mathrm{sec}$; modulation 2500 . The radiofrequency field is perpendicular to the homogeneous magnetic field.

Concentrations between $1 \times 10^{-4}$ and $6 \times 10^{-4} \mathrm{M}$ were used. In the case of cytosine-5-carboxylic acid we used a concentration of $4 \times 10^{-5} \mathrm{M}$. The wavelengths given for the excitation and phosphorescence maxima were corrected for the energy distribution of the light source. The relative intensities could not be measured because it was difficult to adjust the quartz tube with the sample.

Nitro and amino orotic acid showed a weak phosphorescence; we cannot exclude that it may be due to contamination. Bromouracil, azathymine, 5-methyl orotic acid, uracil-6-acetic acid, thymine, dihydro uracil, dihydro thymine, dihydro orotic acid showed no phosphorescence, within reasonable concentrations.

All decay curves, measured at the phosphorescence peak, give a strai fit line in a semilogarithmic plot.

The absorption peaks, presented in Tab. 1, were determined in the solution of dimethyl formamide : ethanol : ether = $1: 1: 1.7$ at room temperature. These absorption bands may approximately represent the envelope of the vibrational structure arising at $77^{\circ} \mathrm{K}$. Diazo uracil, like azo benzene, exhibits three absorption peaks; it may exist in a cis or trans configuration. The absorption peaks of isoorotic acid are concentration and solvent dependent, therefore they are not given in Tab. 1 . With isoorotic acid we were able to demonstrate in preliminary studies that there probably exists a monomer-dimer equilibrium of uracil-5-carboxylic acid via the carboxylic substituent; further studies are in progress. 
Comparison of the excitation peaks with the absorption maxima of the naturally occurring pyrimidine bases (uracil, cytosine) clearly indicates that excitation takes place at the long wavelength part of the corresponding absorption band. This is just the position of the $n-\pi^{*}$ bands.

The excitation and phosphorescence spectrum of uracil is graphed in Fig. 2.

\begin{tabular}{|l|c|c|c|c|l|}
\hline \multicolumn{1}{|c|}{ compound } & EPR & $\begin{array}{c}\text { lifetime } \\
{[\mathrm{sec}]}\end{array}$ & $\begin{array}{c}\text { excitation } \\
{[\mathrm{m} \mu] 77^{\circ} \mathrm{C}}\end{array}$ & $\begin{array}{c}\text { phosphor- } \\
\text { escence } \\
{[\mathrm{m} \mu] 77^{\circ} \mathrm{K}}\end{array}$ & $\begin{array}{l}\text { absorption } \\
{[\mathrm{m} \mu] 22^{\circ} \mathrm{C}}\end{array}$ \\
\hline $\begin{array}{l}\text { orotic acid } \\
\text { orotidine }\end{array}$ & + & 0.20 & 284 & 470 & 280 \\
isoorotic acid & - & 0.25 & 274 & 465 & 268 \\
3- $N$-methyl thymidine & - & -0.1 & 311 & 440 & \\
uracil & - & 0.25 & 270 & 455 & 268 \\
cytosine & - & 0.3 & 294 & 425 & $260 / 298_{\mathrm{S}}$ \\
5-hydroxymethyl cytosine & - & 0.50 & 292 & 424 & 270 \\
cytosine-5-COOH & - & 0.28 & 281 & 417 & 274 \\
2-thiouracil-5-COOH & - & $<0.1$ & 308 & 429 & 279 \\
5-diazo uracil & + & 0.40 & 308 & 500 & $255 / 326$ \\
\hline
\end{tabular}

Tab. 1. Characteristic data of orotic acid and related deriva tives. All measurements were performed in the rigid solvent (dimethyl formamide : ethanol : ether $=1: 1: 1.7)$. The excitation values are corrected wavelenghts. $s$ stands for shoulder.

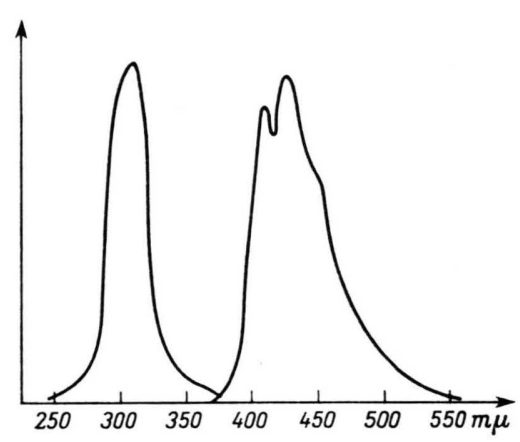

Fig. 2. Uncorrected excitation and phosphorescence spectrum of uracil in the rigid solvent (dimethyl formamide:ethanol: ether $=1: 1: 1.7)$ at $77^{\circ} \mathrm{K}$.

Triplet-triplet energy transfer. To study the triplet-triplet energy transfer between two different molecules we followed the lines of Ermolaev and Terenin ${ }^{7}$. Exciting the $n-\pi^{*}$ transition of benzophenone at about $350 \mathrm{~m} \mu$ there arises an intense and broad phosphorescence band between 430 to $500 \mathrm{~m} \mu$, about. This region thus overlaps the phosphorescence peak of orotic acid at $470 \mathrm{~m} \mu$. Therefore it was to be hoped that the donor triplet of benzophenone might be transferred to orotic acid as acceptor molecule by an exchange mechanism.

Benzophenone $\left(5 \times 10^{-3} \mathrm{M}\right)$ and orotic acid $\left(8 \times 10^{-2} \mathrm{M}\right)$ were mixed in our standard rigid solvent. The degassed sample was put in the micro-

7 V. L. Ermolaev and A. Terenin, J. Chim. physique 55, 698 [1958]. wave cavity of the EPR spectrometer. Cutting off light below $350 \mathrm{~m} \mu$, the orotic acid triplet signal could be detected. Doing the same experiment in the absence of benzophenone, no triplet signal appeared. Furthermore, the triplet state of benzophenone could not be recorded by EPR-techniques.

Photochemistry. As previously reported ${ }^{5}$, irradiation of an aqueous solution of orotic acid produces orotic acid dimer. The cyclobutane ring structure of the dimer can be split by $240 \mathrm{~m} \mu$ radiation. Preliminary studies of orotic acid in alcoholic solution show a distinct solvent dependence of the photochemical reaction. Maximal irradiation of orotic acid $\left(2 \times 10^{-4} \mathrm{M}, 22{ }^{\circ} \mathrm{C}\right)$ in ethanol with the light of a Osram HB 200 lamp lowers the absorption peak at $283 \mathrm{~m} \mu$ to about 5 per cent of its original height. At the same time there arises a pronounced peak at about $253 \mathrm{~m} \mu$. Compared with an aqueous solution there is no end-absorption spectrum as in the case of orotic acid dimer. This seems to indicate that water is somehow involved in the dimerization reaction.

\section{Discussion}

The EPR triplet state signal of orotic acid is characterized by a large zero-field splitting because of $\mathrm{H}_{\min }=1232 \mathrm{G}$. The zero-field splitting constant is calculated as $D=0.22 \mathrm{~cm}^{-1}$ according to the relation given by de Groot and van Der WaALs ${ }^{6}$. We did not find $\Delta \mathrm{M}=1$ transitions in our randomly oriented molecules. 
This triplet state electron enhances the spin density of the 5,6-double bond, which increase is necessary for a dimerization reaction ${ }^{4}$. Experiments, previously reported, showed a pronounced oxygen effect in the photochemical dimerization of orotic acid in aqueous solution ${ }^{5}$. Thus, we can conclude that the triplet state is responsible for dimerization. But besides the triplet state, the surrounding medium plays some active role in this photochemical process.

With orotic acid the EPR triplet signal practically disappeared when increasing the temperature from $77^{\circ} \mathrm{K}$ to about $95^{\circ} \mathrm{K}$. Compared to the long lifetime of the triplet state, the relaxation times of the solute environment are probably still short enough to equilibrate the initial orientation. Thus, the rigidity of the glassy matrix, or the initial orientation of its molecules, influences critically the triplet state.

Besides diazo uracil, it was impossible to observe EPR absorption from the phosphorescent triplet state of the other compounds tested. This fact may be due to large zero-field splitting, which is expected from the N-heterocyclics ${ }^{8}$. Thomson ${ }^{9}$ measured pyrazine in Lucite without detecting an EPR triplet signal. He attributed this failure to the short

8 C. A. Hutchison and B. W. Mangum, J. chem. Physics 34, 908 [1961].

9 C. Thomson, J. chem. Physics 41, 1 [1964]. lifetime of the $n-\pi^{*}$ triplet $(\approx 0.03 \mathrm{sec})$ or to zero-field splitting. Of course, it might be successful to use instead of $3 \mathrm{~cm}$ microwaves shorter waves or a memory EPR spectrometer, respectively.

The excitation peak of uracil and cytosine distinctly differs from the corresponding absorption peak. It may be attributed to a $n-\pi^{*}$ excitation because it lies at the long wavelength side of the absorption peak ${ }^{2}$. Lifetimes greater than $0.1 \mathrm{sec}$ are due to the decay of a $\pi-\pi^{*}$ triplet level; the generally known lifetimes of $n-\pi^{*}$ triplets are of the order of $10^{-2} \mathrm{sec}$ or $10^{-3} \mathrm{sec}$, respectively.

In the case of pyrimidine, Loustauneau et al. ${ }^{10}$ found that the population of the $\pi-\pi^{*}$ triplet level occurs via excitation of a $n-\pi^{*}$ singlet level in the following way. This $n-\pi^{*}$ singlet state undergoes internal conversion to a $n-\pi^{*}$ triplet state, and from the latter the lower lying $\pi-\pi^{*}$ phosphorescent triplet level is populated. A similar process may occur with cytosine.

Bersohn and Isenberg ${ }^{11}$ did not find a measurable phosphorescence of thymine, cytosine, and uracil, in the cracking water-glycerol mixture at $77^{\circ} \mathrm{K}$.

10 P. Loustauneau, G. Nouchi and A. Rousset, C. R. Acad. Sci. Paris 257, 2928 [1963].

11 R. Bersohn and J. Isenberg, J. chem. Physics 40, 3175 [1964]. 